\title{
ANÁLISIS DE LOS RECURSOS PARA LA ENSEÑANZA DE LECTOESCRITURA INICIAL A LA LUZ DEL CURRÍCULO DOMINICANO
}

\section{Analysis of Resources for the Teaching of Early Literacy in the Light of the Dominican Curriculum}

\author{
Ramón Marcelino Viñas-Marte ${ }^{1}$ \\ rvinas1965@gmail.com
}

Lourdes Natalia Guzmán-Taveras ${ }^{1}$

Inataliaguzman@gmail.com

\section{Resumen}

Los recursos didácticos utilizados para la enseñanza de la lectoescritura inicial (LEI) son herramientas que acercan a los estudiantes a la cultura letrada y permiten el reconocimiento del código escrito y sus características. La alineación de los recursos pedagógicos con lo prescrito en el currículo de enseñanza puede influir positivamente en el rendimiento de la lectoescritura en la República Dominicana, uno de los más bajos de la región. En este sentido, surge el interés de analizar las características de los recursos pedagógicos de la base de datos de lectoescritura inicial LEI-RD de acceso libre. Se diseñó un estudio mixto con el objetivo de determinar la alineación de los recursos de dicha base con el currículo dominicano vigente e identificar áreas de mejora. Los recursos se analizaron a partir de las siguientes dimensiones: finalidad, habilidades de la LEI, enfoque de enseñanza, animación lectora y pertinencia del recurso. Se seleccionaron aleatoriamente 115 recursos de un total de 485. Los resultados muestran que la mayoría de los recursos proponen intervenciones acordes con los enfoques de enseñanza del currículo dominicano: el enfoque textual, funcional y comunicativo, y el enfoque procesual cognitivo por competencias. Se destaca la carencia de actividades destinadas a los niveles bajos de procesamiento lector y a la animación lectora. Se sugiere la revisión de los criterios de inclusión de los recursos que integran la base LEI-RD para garantizar la alineación con la evidencia actual sobre la enseñanza efectiva de la LEI y el currículo dominicano vigente.

Palabras clave: currículo, Educación Primaria, escritura, lectura, recursos educativos.

\section{Abstract}

The didactic resources used for teaching Early Grade Literacy (EGL) are tools that bring students closer reading culture, allowing them to recognize written code and its characteristics. The alignment of pedagogical resources with what is prescribed in the teaching curriculum has positive influence on literary performance, which in the Dominican Republic, is reported as one of the region's lowest. Due to this, there is an interest in analyzing the characteristics of the pedagogical resources in the EGL free-access database (LEI$\mathrm{RD})$. A mixed study was designed with the objective of determining the alignment of said database with the current Dominican curriculum, and identifying areas of improvement. A total of 115 pedagogical resources were randomly choosen from the 485 resources in the database. The results showed that the majority of resources propose interventions in line with the teaching approaches that underpin the Dominican curriculum: the textual, functional, and communicative approach, as well as the cognitive process by competencies. It is suggested that the inclusion criteria of the resources that make up the LEI-RD database be reviewed to ensure alignment with current evidence on the effective teaching of the LEI and the current Dominican curriculum.

Keywords: curriculum, reading, writing, Elementary Education, educational resources. 


\section{Introducción}

En la República Dominicana, la enseñanza de la lectoescritura inicial (LEI) dista de lo que plantean los enfoques basados en evidencia que orientan el aprendizaje de la lengua escrita. El currículo dominicano promueve el enfoque centrado en la construcción del significado y la comunicación. En este subyace el enfoque procesual cognitivo por competencia; el textual, funcional y comunicativo y el sociocultural; influidos por las teorías de Piaget y Vygotsky y a través de los cuales se abordan la lectura y la escritura como procesos y prácticas sociales (Ministerio de Educación de la República Dominicana [MINERD], 2016).

Sin embargo, la transposición didáctica en muchas de las aulas de primaria evidencia la implementación de estrategias y actividades en las que subyacen enfoques incoherentes con el currículo aceptado a nivel nacional. Además, al parecer, muchos de los recursos pedagógicos utilizados para la enseñanza y aprendizaje de la LEI no están alineados con dicho currículo. Estos promueven el uso de la repetición sin compresión basados en enfoques por destrezas, los cuales ponen énfasis en la enseñanza de unidades lingüísticas aisladas y descontextualizadas (Montenegro, 2013). Cambiar este panorama y contar con material didáctico adecuado al contexto y alineado a la evidencia actual de la enseńanza efectiva de la LEI es uno de los mayores retos para la República Dominicana (Vijil, 2019).

Los resultados de estudios nacionales e internacionales que miden la competencia lectora de los estudiantes al finalizar el tercer grado y el primer ciclo de la Educación Primaria colocan a la República Dominicana en los últimos lugares a nivel de comprensión lectora, en relación con la media regional (MINERD, 2017). Según estos resultados, solo un $46 \%$ de los estudiantes de tercer grado alcanza el nivel I de desempeño correspondiente a una comprensión literal del texto, y solo el $12 \%$ de los estudiantes posee las competencias comunicativas que establece el currículo dominicano.

Con relación a la forma de enseñanza en el país, De Lima (2018) analizó las concepciones de lectura y es- critura que tienen los alfabetizadores en la República Dominicana, su correspondencia con la práctica pedagógica y enfoques de enseñanza. Los resultados de esta investigación indican que en la teoría el 33.7\% de los docentes afirma basar sus intervenciones en el constructivismo, mientras que en la práctica ese porcentaje se redujo al $0.90 \%$.

Teniendo presente esta realidad, en 2019 comienza la colaboración del Programa de Capacidades LAC Reads (PCLR) con la Pontificia Universidad Católica Madre y Maestra (PUCMM) para elaborar una base de datos de recursos y evidencias (creados por instituciones públicas, privadas y sin fines de lucro en el país) sobre la LEI en la República Dominicana (LEIRD) con el objetivo de facilitar a actores interesados el acceso a material que promueva la comprensión y aplicación de enfoques basados en la evidencia y en el contexto apropiado para mejorar las competencias de la LEI en el país, así como contribuir en la toma de decisiones de las políticas públicas sobre el tema. Partiendo de lo descrito anteriormente, interesa responder las siguientes preguntas: ¿Cómo se alinean los recursos de LEI-RD con el currículo del primer ciclo de primaria? ¿Cuál es el enfoque de enseńanza que predomina en los recursos pedagógicos incluidos en LEI-RD?

Por tal razón se diseñó esta investigación con el objetivo de analizar las características de los recursos de la base de datos LEI-RD, ya que se desconoce si se encuentran alineados a lo prescrito en el currículo dominicano vigente. A partir del análisis se identificaron las áreas de mejora de los recursos que la conforman y de su estructura.

\section{Revisión de la literatura}

\subsection{Base de datos de lectoescritura inicial de la República Dominicana LEI-RD}

La base de datos LEI-RD se presentó públicamente en septiembre de 2019; actualmente está conformada por 485 recursos pedagógicos, 427 documentos teóricos y 26 evidencias sobre el tema de la LEI, desde el nacimiento hasta el tercer grado de primaria, producidos desde el año 2005 hasta el 2019 y que 
cuentan con la autorización de las organizaciones que los han elaborado para ser de acceso público. La base de datos LEI-RD está disponible en línea para el uso de académicos, investigadores, docentes, instituciones gubernamentales - tales como el Ministerio de Educación y el Ministerio de Educación Superior, Ciencia y Tecnología-, las ONG y cooperantes nacionales e internacionales.

Esta base cuenta con dos secciones: una contiene 26 evidencias registradas en revistas científicas y la otra incluyen los recursos y documentos teóricos utilizados en la enseńanza y el aprendizaje de la LEI. Con relación a los 427 documentos teóricos, se consideran los siguientes: tesis o disertaciones, evaluaciones de programas, publicaciones internas, folletos, documentos de mejores prácticas, informes, libros autopublicados y documentos normativos (leyes, directrices, planes estratégicos, etc.), entre otros. Entre sus 485 recursos pedagógicos figuran los siguientes: ayudas visuales, audios, videos, libros de texto, cuadernos de trabajo, guías para los docentes, planes de estudios, currículos, instrumentos de evaluación, y material de apoyo para la formación docente, entre otros.

Después de una revisión exhaustiva de la literatura, no se han encontrado evidencias similares dedicadas al estudio de la calidad de los recursos pedagógicos destinados a desarrollar habilidades de la LEI. Otros países de América Central y el Caribe, bajo la iniciativa del PCLR, también han elaborado bases de datos con los recursos que utilizan, sin embargo, ninguno ha analizado con base científica la calidad de los mismos. De acuerdo con esta revisión, este estudio se considera pionero en el campo de investigación.

\subsection{Enseñanza de la lectoescritura en el Primer ciclo del Nivel Primario $\left(1 .^{\circ}, 2 .^{\circ}\right.$ y $3 .^{\text {er }}$ grado) de la República Dominicana}

A partir de la revisión curricular establecida por el MINERD en el 2016, el plan de estudio oficial del primer ciclo de la educación primaria establece que el enfoque centrado en la construcción del significado y en la comunicación debe influir las prácticas de enseñanza y aprendizaje de la LEI. Según Montenegro (2013) este enfoque:
Asume la lectura y la escritura como procesos de construcción y de expresión de significados. Se reconoce la función social y comunicativa de la lengua escrita y su unidad fundamental es el texto para desarrollar las capacidades lingüísticas de los sujetos. La enseñanza de la lectura y la escritura es siempre guiada por unos propósitos definidos y destinatarios reales, tal como ocurre en la vida. La teoría epistemológica que fundamenta este paradigma es la constructivista y su enfoque es sociocultural al entender que se aprende en constante interacción con el medio, las personas y los objetos (pp. 7-8).

El currículo también se apoya en las evidencias psicogenéticas que han dejado al descubierto la manera en que los nińos se aproximan al sistema de escritura y a la lengua escrita cuando leen y escriben (Ferreiro, 2006; Ferreiro \& Teberosky, 1991; Fons, 2004; Kaufman, 2009; Teberosky, 2000). Según estas evidencias, la evolución de la escritura de los niños experimenta diversas fases: comienza con el dibujo como portador de significado, sigue con la utilización de letras o signos para representar sonidos, hasta que accede al principio alfabético. En este enfoque se da mucha importancia al pensamiento infantil, por lo que se parte de los intereses y saberes previos de los estudiantes. Las características del sistema de escritura y el entorno adquieren un valor relevante en el desarrollo de las competencias lingüísticas del estudiantado en los primeros ańos de la educación.

La estructura del currículo de Lengua Española se apoya en un enfoque cognitivo basado en competencias. Establece cuatro competencias específicas, a saber: comprensión oral, producción oral, comprensión escrita y producción escrita. Entiende la competencia como "la capacidad para actuar de manera eficaz y autónoma en contextos diversos movilizando de forma integrada conceptos, procedimientos, actitudes y valores" (MINERD, 2016, p. 34). De estas competencias se derivan diversas habilidades que se articulan en la propuesta curricular. La comprensión y producción oral propone el desarrollo de procedimientos propios de conciencia fonológica, para que los aprendices tengan la oportunidad de reconocer 


\section{VIÑAS-MARTE • GUZMÁN-TAVERAS}

Análisis de los recursos para la enseñanza de lectoescritura inicial a la luz del currículo dominicano

que el habla se compone de unidades sencillas y manipulables llamadas fonemas. La producción oral también contempla la fluidez al expresarse oralmente sobre hechos, ideas y acontecimientos con velocidad y entonación adecuadas.

Por otro lado, las demás competencias, comprensión escrita y producción escrita, promueven el contacto con el código, llevando al estudiante de la oralidad a su representación. En la comprensión escrita se proponen procedimientos de decodificación, los cuales establecen la relación sonoro-gráfica y propician el paso hacia la adquisición de la lengua escrita. También la fluidez lectora, entendida como la capacidad de leer cualquier texto de forma precisa, a buen ritmo y con entonación adecuada, es un aspecto importante en el desarrollo de la comprensión. Tanto la producción escrita como la comprensión escrita abarcan las convenciones de la lectoescritura, es decir, la direccionalidad, la linealidad y la adecuada disposición del escrito sobre el papel.

El desarrollo eficaz de estas habilidades, trabajadas sobre el texto, acercan al logro del objetivo macro: la manifestación de competencias lectoras y escriturales. Por medio de estos procedimientos se propicia el descubrimiento del principio alfabético, un paso esencial para el desarrollo de la comprensión lectora y el alcance de aprendizajes que permitan asumir la lectura y la escritura, como plantea el enfoque centrado en la construcción de significado y la comunicación, es decir, como prácticas sociales guiadas por una intención comunicativa.

En el primer ciclo de la Educación Primaria se propone la mayor diversidad de situaciones de LEI para garantizar el contacto de los estudiantes con textos escritos para su exploración, selección y lectura. Además, para lograr que ellos produzcan textos o escritos diversos con variadas intenciones, sobre temas de su interés y dirigidos a destinatarios distintos. Se busca que en cada grado de este ciclo los estudiantes se sientan lectores y escritores de textos como prácticas habituales de comunicación y reflexión de su aprendizaje (MINERD, 2016, p. 77).

Estas sugerencias guardan relación con el enfoque centrado en la construcción de significado y la co- municación, pues prioriza el uso de la lengua en contextos reales, siendo el texto la unidad base, pues los seres humanos no se comunican mediante palabras aisladas. Para ajustar la enseñanza a esta realidad, es imprescindible que los estudiantes lean y produzcan textos considerando un propósito claro, el destinatario, el contexto y otros elementos que intervienen en la situación comunicativa.

El currículo dominicano sugiere diversificar el uso de recursos para favorecer el desarrollo de las competencias y acercar a los nińos a la cultura escrita:

Se recomienda integrar al aula materiales impresos, que son aquellos que utilizan principalmente códigos verbales y gráficos, tales como libros, guías de estudio, fichas de trabajo, ejercicios prácticos, láminas e imágenes. Las aulas han de ser letradas de forma acorde con las temáticas a trabajar, contar con recursos para el acercamiento de los niños y las niñas a la cultura escrita: caja de palabras clasificadas, tarjetas para asociar imágenes y palabras, variedad de textos, alfabetos móviles y fijos, nombres y apellidos de cada niño y niña y todo tipo de recursos que sirvan para motivar el interés por la lectura y la escritura (MINERD, 2016, pp. 4849).

En efecto, el currículo dominicano adopta el enfoque centrado en la construcción de significado y en la comunicación, el cual propicia situaciones en las que se evidencie el uso funcional de la lengua. En este marco se valora el contacto con diversos textos y el uso de recursos pedagógicos que acerquen a los niños a la cultura escrita.

\subsection{Panorama de la evidencia relacionada con los recursos utilizados en la enseńanza y aprendizaje de la LEI}

La revisión de la literatura demuestra la escasez de evidencias sobre la enseńanza y aprendizaje de la LEI en Latinoamérica y el Caribe. Las brechas se relacionan con la baja calidad de los estudios que se producen en la región, incluyendo las publicaciones sobre el desarrollo de habilidades de preescritura y escritura y su relación con los resultados de la LEI en pruebas nacionales e internacionales y en investigaciones relacionadas con dicho tema (Stone, 2019). 
Otros estudios referidos a la enseñanza y aprendizaje de la LEI muestran la necesidad de utilizar recursos pedagógicos destinados a la alfabetización (Kim et al., 2016). Se indica que los recursos pedagógicos dedicados a la enseńanza y el uso de libros de texto desempeñan un rol esencial en la adquisición de la lengua escrita; además, se requiere el contacto con textos para desarrollar las convencionalidades de la LEI y la conciencia del código escrito.

De Lima y Montenegro (2010) subrayan la importancia de utilizar recursos pedagógicos en contextos socioculturales vulnerables, en los que los niños tienen escaso contacto con la cultura escrita en sus hogares y en la comunidad. Es preciso tomar en cuenta las conceptualizaciones de los niños acerca de qué es leer y escribir, las características del sistema de escritura y la práctica docente. Por eso, en los materiales didácticos (guías de enseñanza y aprendizaje), según De Lima y Montenegro (2010), se deben incluir actividades relacionadas con el todo textual, con las partes de texto y con el uso funcional del lenguaje. De esta manera, los recursos pedagógicos parten de tipologías textuales usadas en la vida diaria, sin abandonar sus partes (letras, palabras, sonidos, ortografía, vocabulario y otros elementos). Estas características enunciadas por las autoras guardan correspondencia con el currículo dominicano, el cual propone en cada unidad partir de un texto desde el que se aborda la conciencia fonológica, la decodificación y los demás aspectos lingüísticos.

Por otra parte, las guías didácticas constituyen otro valioso recurso pedagógico que aporta al docente que no ha recibido formación requerida para enseñar de forma efectiva la LEI, un soporte que le permita generar situaciones de aprendizaje para que los niños comprendan el sistema de escritura (De Lima \& Montenegro, 2010). Sin embargo, según las autoras, ese carácter de apoyo puede constituirse en una gran debilidad si en la capacitación no se prevén estrategias para que gradualmente el docente haga modificaciones según los requerimientos del estudiantado.

Los niños y niñas desarrollan habilidades que favorecen la adquisición de la LEI. Entre estas se encuentra la conciencia fonológica, la fonética, el vocabulario, la fluidez y la comprensión lectora (National Reading Panel, National Institute of Child Health and Human Development, 2000). Al respecto, Gutiérrez y Díez (2018) indagan la relación entre la conciencia fonológica y el desarrollo evolutivo de la escritura, y los resultados aseveran que:

Existe una correlación directa y significativa entre el aprendizaje de la escritura y las distintas habilidades que facilitan la toma de conciencia de las unidades mínimas que componen las palabras (sílabas, unidades intrasilábicas y fonemas), lo que indica que la capacidad de manejar y reflexionar sobre las unidades del lenguaje hablado es de gran relevancia para la facilitación del aprendizaje de la producción escrita en las primeras edades (p. 405).

En las evidencias consultadas y el currículo vigente están presentes las siguientes dimensiones, las cuales se describen brevemente:

1. La finalidad o propósitos se refiere al grupo que desarrollará la habilidad o competencia a la que está destinado cada recurso según su contenido y lo descrito en LEI-RD (MINERD, 2016; Ministerio de Educación de Argentina, 2010; Romero, 2014).

2. Las habilidades de la LEI propias del proceso de adquisición (Daviña, 2003; Ferreiro \& Teberosky, 1991; Kaufman, 2009; Ortiz \& Robino, 2003; Rasinski, 2010; Rosemberg et al., 2014; Shanahann, 2005);

3. Los enfoques de enseñanza, que son ideas sobre lo que es enseñado y lo que debería ser enseñado (Kaufman, 2009; MINERD, 2016; Montenegro, 2010);

4. La animación lectora, concebida como la acción dirigida a crear un vínculo entre un material de lectura y un individuo o grupo (Blanco et al., 2007; Fons, 2004; Charria \& González, 1992; Kaufman, 2009; MINERD, 2016; Ministerio de Educación de Chile, 2011; Yepes et al., 2013). 


\section{VIÑAS-MARTE • GUZMÁN-TAVERAS}

Análisis de los recursos para la enseñanza de lectoescritura inicial a la luz del currículo dominicano

\section{Metodología}

Se diseñó un estudio mixto de carácter exploratorio con alcance descriptivo. El objetivo fue analizar las características de los recursos de la base de datos LEI-RD para la enseñanza de la LEI a la luz del currículo dominicano vigente del primer ciclo de primaria. Esta investigación se realizó desde octubre de 2019 hasta marzo de 2020. Debido al tiempo disponible para realizar la investigación, se conformó una muestra aleatoria de 115 recursos pedagógicos de los 485 presentes en la base.

Para responder a las siguientes preguntas de investigación: ¿Cómo se alinean los recursos de LEI-RD con el currículo del primer ciclo de primaria? y ¿Cuál es el enfoque de enseñanza que predomina en los recursos pedagógicos incluidos en LEI-RD? se realizaron diversos procedimientos, a saber: exploración de la base de datos, determinación de las dimensiones, categorías y subcategorías; análisis de los recursos pedagógicos, categorización de los recursos según su pertinencia, tabulación de los resultados e interpretación de los hallazgos. Estos procedimientos se realizaron en tres fases, las cuales se describen a continuación.

En la primera fase se examinó la base de datos: se observó detenidamente la estructura, que comprende dos grandes grupos: los recursos pedagógicos y los documentos teóricos. Durante la exploración se identificaron puntos de encuentro y divergencias entre las características de los recursos destinados al primer ciclo de primaria y el currículo. Esto orientó la definición de las categorías más relevantes para el análisis, de acuerdo con la naturaleza de los recursos y la revisión de la literatura. Asimismo, se identificaron y se describieron las instituciones autoras de dichos recursos, tal como se muestra en la Tabla 1.

Tabla 1. Tipos de instituciones autoras de los recursos

\begin{tabular}{|c|c|c|}
\hline Tipos de institución & Ejemplos & Definición \\
\hline Públicas & $\begin{array}{l}\text { - Ministerio de Educación } \\
\text { (MINERD) } \\
\text { - Instituto Superior de Formación } \\
\text { Docente Salomé Ureña } \\
\text { (ISFODOSU) }\end{array}$ & $\begin{array}{l}\text { Instituciones financiadas por el Estado, con per- } \\
\text { sonal pagado por el Estado. }\end{array}$ \\
\hline Privadas & $\begin{array}{l}\text { - Pontificia Universidad Católica } \\
\text { Madre y Maestra (PUCMM) } \\
\text { - Universidad Iberoamericana } \\
\text { (UNIBE) } \\
\text { - Instituto Tecnológico de Santo } \\
\text { Domingo (INTEC) }\end{array}$ & $\begin{array}{l}\text { Entidades educativas financiadas por el sector } \\
\text { privado y cuyo objetivo principal es la producción } \\
\text { y difusión del conocimiento. }\end{array}$ \\
\hline Instituciones sin fines de lucro & $\begin{array}{l}\text { - Organización de Estados Ibe- } \\
\text { roamericanos (OEI) } \\
\text { - } \text { Save the Children } \\
\text { - } \text { Agencia de los Estados Unidos } \\
\text { para el Desarrollo Internacional } \\
\text { (USAID) } \\
\text { - DREAM Project } \\
\text { - Plan Internacional } \\
\text { - Centro Cultural Poveda } \\
\text { - Centro de Excelencia para } \\
\text { la Capacitación de Maestros } \\
\text { (CETT) }\end{array}$ & $\begin{array}{l}\text { Organizaciones nacionales e internacionales que } \\
\text { realizan o colaboran con programas educativos } \\
\text { en el país. }\end{array}$ \\
\hline
\end{tabular}

Nota: Elaborado a partir de la base de datos LEI-RD. 
En esta fase también se determinó la cantidad de recursos analizados según la institución autora que los produjo. Además, se muestra cuántos recursos son de libre acceso o descargables y cuántos se consultan en línea. La mayoría de los recursos presentes en la muestra pertenecen a instituciones públicas, como el Ministerio de Educación. Otra parte está concentrada en instituciones privadas como la Universidad
Iberoamericana, el Centro de Excelencia para la Investigación y Difusión de la Lectura y Escritura de la Pontificia Universidad Católica Madre y Maestra (PUCMM-CEDILE) y el Instituto Tecnológico de Santo Domingo (INTEC), y el resto corresponde a instituciones sin fines de lucro, tal como se muestra en la Tabla 2.

Tabla 2. Recursos de LEI-RD. Cantidad por institución: descargables y en línea

\begin{tabular}{lcccc}
\hline & Recursos por institución & & \\
\hline \multicolumn{1}{c}{ Institución } & FRE $(\boldsymbol{n}=\mathbf{1 1 5})$ & $\%$ & R. DESC. & R. E. Lí \\
\hline Universidad Iberoamericana & 12 & $10.40 \%$ & 12 & 0 \\
PUCMM-CEDILE & 15 & $13.04 \%$ & 15 & 0 \\
CETT & 15 & $13.04 \%$ & 15 & 0 \\
CEED-INTEC & 2 & $1.74 \%$ & 2 & 0 \\
Plan Internacional & 1 & $0.87 \%$ & 1 & 0 \\
Ministerio de Educación & 45 & $39.13 \%$ & 37 & 8 \\
ISFODOSU & 1 & $0.87 \%$ & 1 & 0 \\
Save the Children & 8 & $6.96 \%$ & 8 & 0 \\
Dream Project & 6 & $5.22 \%$ & 6 & 0 \\
Organización Estados Iberoamericanos & 5 & $4.35 \%$ & 5 & 0 \\
Centro Cultural Poveda & 5 & $4.35 \%$ & 5 & 0 \\
Total & $\mathbf{1 1 5}$ & $\mathbf{1 0 0 . 0 0 \%}$ & $\mathbf{1 0 7}$ & $\mathbf{8}$ \\
\hline
\end{tabular}

Nota: Elaborado a partir de la base de datos LEI-RD. $n=115$ : muestra seleccionada. (FRE) Frecuencia; (R. DESC.) Recurso descargable; (R.E.LÍ.) Recurso en línea. PUCMM: Pontificia Universidad Católica Madre y Maestra; CEDILE: Centro de Excelencia para la Investigación y Difusión de la Lectura y la Escritura; CEED-INTEC: Centro de Estudios Educativos; ISFODOSU: Instituto Superior de Formación Docente Salomé Ureña. CETT: Centros de Excelencia para la Capacitación de Maestras y Maestros de Centroamérica.

A partir del análisis anterior se elaboró el árbol de dimensiones, categorías y subcategorías utilizadas para el estudio de los recursos. Se consideraron dos fuentes claves de información: lo propuesto por el National Reading Panel, National Institute of Child Health and Human Development (2000) y el sustento teórico del currículo dominicano (MINERD, 2016). Las dimensiones seleccionadas fueron las siguientes: 1. Finalidad, la cual se refiere al grupo que desarrollará la habilidad o competencia a la que está destinado cada recurso según su contenido y lo descrito en LEI-RD; 2. Habilidades de la LEI propias del proceso de adquisición; 3. Enfoques de enseñan- za, conceptualizados como las ideas sobre lo que es enseñado y lo que debería ser enseñado; y 4. Animación lectora, concebida como la acción dirigida a crear un vínculo entre un material de lectura y un individuo o grupo. De cada una de las dimensiones se derivan varias categorías y subcategorías que permitieron la codificación de los recursos estudiados y están determinadas a partir de la exploración de los recursos de LEI-RD, la consulta de las evidencias y el currículo (Tabla 3).

Se consideraron los componentes curriculares mencionados anteriormente para determinar la alinea- 


\section{VIÑAS-MARTE • GUZMÁN-TAVERAS}

Análisis de los recursos para la enseñanza de lectoescritura inicial a la luz del currículo dominicano

ción de los recursos con lo propuesto en el currículo. Durante el análisis se examinó la presencia de las competencias y habilidades que propone este docu- mento, así como aquellas que evidencian resultados de buenas prácticas en la literatura mencionada anteriormente.

Tabla 3. Dimensiones, categorías y subcategorías para el análisis de los recursos de la base de datos LEI-RD

\begin{tabular}{|c|c|c|}
\hline \multirow{2}{*}{$\frac{\text { Dimensiones }}{1 \text {. Finalidad del recurso }}$} & Categorías & Subcategorías \\
\hline & $\begin{array}{l}\text { 1.1. Aprendizaje de la lectura } \\
\text { 1.2. Enseñanza de la escritura } \\
\text { 1.3. Aprendizaje de la lectura } \\
\text { 1.4. Enseñanza de la escritura } \\
\text { 1.5. Evaluación de los aprendizajes } \\
\text { 1.6. Formación docente } \\
\text { 1.7. Acompañamiento pedagógico }\end{array}$ & $\begin{array}{l}\text { 1.5.1. Diagnóstica } \\
\text { 1.5.2. Formativa } \\
\text { 1.5.3. Sumativa }\end{array}$ \\
\hline 2. Habilidades de la lectoescritura & $\begin{array}{l}\text { 2.1. Comprensión oral } \\
\text { 2.2. Producción oral } \\
\text { 2.3. Conciencia fonológica } \\
\text { 2.4. Comprensión escrita } \\
\text { 2.5. Convenciones de la lectoescritura } \\
\text { 2.6. Decodificación } \\
\text { 2.7. Vocabulario } \\
\text { 2.8. Fluidez lectora } \\
\text { 2.9. Producción escrita } \\
\text { 2.10. Etapas de apropiación de la } \\
\text { escritura } \\
\text { 2.11. Dominio del código escrito }\end{array}$ & $\begin{array}{l}\text { 2.11.1. Transcripción } \\
\text { 2.11.2. Caligrafía }\end{array}$ \\
\hline 3. Enfoques de enseñanza & $\begin{array}{l}\text { 3.1. Textual, funcional y comunicativo } \\
\text { 3.2. Procesual cognitivo, por competen- } \\
\text { cia } \\
\text { 3.3. Sociocultural } \\
\text { 3.4-Por destrezas aisladas }\end{array}$ & \\
\hline 4. Motivación lectora & $\begin{array}{l}\text { 4.1. Ambiente letrado } \\
\text { 4.2. Promoción del hábito lector } \\
\text { 4.3. Biblioteca áulica y escolar } \\
\text { 4.4. Familia y hábito lector } \\
\text { 4.5. Escuela y hábito lector }\end{array}$ & \\
\hline
\end{tabular}

Nota: Elaboración propia a partir de la base de datos LEI-RD. Se revisó al currículo dominicano de Lengua Espańola y otros textos.

La segunda fase consistió en el análisis de recursos pedagógicos a través de la lectura comprensiva de su contenido. Estos recursos fueron analizados de acuerdo con las categorías mencionadas. Para la organización de los datos recolectados se utilizó el software NVivo y en la sistematización y análisis se utilizó una lista de cotejo tipo Likert diseñada por los autores, que contempla los aspectos a evaluar y la escala de estimación elaborada a partir de la literatura consultada (Tabla 4). 
Revista Caribeña de Investigación Educativa | 2020, 4(2), 96-111

Tabla 4. Escala de valoración del instrumento utilizado (lista de cotejo)

\begin{tabular}{|c|c|c|c|}
\hline $\begin{array}{l}\text { Escala de valo- } \\
\text { ración (lista de } \\
\text { cotejo) }\end{array}$ & Valor & $\begin{array}{l}\text { Rango de cla- } \\
\text { sificación del } \\
\text { recurso }\end{array}$ & Significado \\
\hline Satisfactorio & 4 & 3.1 a 4 & $\begin{array}{l}\text { El recurso es coherente con los enfoques y teorías que plan- } \\
\text { tea el currículo y presenta actividades que orientan el proceso } \\
\text { de enseñanza y aprendizaje de la LEI. Cumple con el objetivo } \\
\text { para el que fue diseñado y se ajusta al grupo para el que fue } \\
\text { diseñado. }\end{array}$ \\
\hline $\begin{array}{l}\text { Medianamente } \\
\text { satisfactorio }\end{array}$ & 3 & 2.1 a 3 & $\begin{array}{l}\text { El recurso coincide con los lineamientos establecidos en el } \\
\text { currículo, pero solo integra algunas de las habilidades de la } \\
\text { enseñanza y aprendizaje de la LEl. Responde parcialmente } \\
\text { al propósito para el que fue diseñado y a las necesidades del } \\
\text { grupo al que fue dirigido }\end{array}$ \\
\hline $\begin{array}{l}\text { Área de oportuni- } \\
\text { dad }\end{array}$ & 2 & 1.1 a 2 & $\begin{array}{l}\text { El recurso promueve actividades de la LEI poco coherentes } \\
\text { con el currículo. Las intervenciones pedagógicas que propone } \\
\text { están desvinculadas con lo que muestran las evidencias. Ob- } \\
\text { via aspectos fundamentales sobre la adquisición de la lengua } \\
\text { escrita }\end{array}$ \\
\hline No satisfactorio & 1 & 1 & $\begin{array}{l}\text { El recurso no es coherente con los lineamientos curriculares } \\
\text { ni con las evidencias. Se desvía de la finalidad de la base de } \\
\text { datos LEI-RD. }\end{array}$ \\
\hline
\end{tabular}

Nota: Para clasificar los recursos se obtuvo un promedio, se sumaron los puntos obtenidos en el instrumento de evaluación y se dividieron entre el número de respuestas marcadas.

En la última fase se tabularon los resultados y se representaron en tablas los datos más significativos, para facilitar el análisis de los hallazgos, realizar inferencias y determinar el enfoque predominante en el contenido de los recursos pedagógicos de LEI-RD y su alineación con el currículo. La Tabla 5 resume las herramientas que se utilizaron en esta investigación para responder las preguntas planteadas.

Tabla 5. Preguntas de investigación, fuentes, muestra y métodos de recolección

\begin{tabular}{llll}
\hline Preguntas de investigación & Fuente & Muestra & Método de recolección \\
\hline $\begin{array}{l}\text { ¿Cuál es el enfoque de enseñanza que } \\
\text { predomina en el contenido de los recur- } \\
\text { sos pedagógicos incluidos en la base de } \\
\text { datos de lectoescritura inicial LEI-RD? }\end{array}$ & $\begin{array}{l}\text { Lectura explora- } \\
\text { toria y análisis de } \\
\text { los recursos }\end{array}$ & $\begin{array}{l}115 \\
\text { recursos }\end{array}$ & $\begin{array}{l}\text { Exploraron de los recursos de la base } \\
\text { de datos LEl-RD }\end{array}$ \\
\hline $\begin{array}{l}\text { ¿Cómo se vinculan los recursos de LEl- } \\
\text { RD con el currículo del primer ciclo de } \\
\text { primaria y con las evidencias sobre el } \\
\text { tema? }\end{array}$ & $\begin{array}{l}\text { Análisis y clasi- } \\
\text { ficación de los } \\
\text { recursos }\end{array}$ & $\begin{array}{l}115 \\
\text { recursos }\end{array}$ & $\begin{array}{l}\text { Establecimiento de dimensiones, cate- } \\
\text { gorías y subcategorías }\end{array}$ \\
\hline
\end{tabular}

Nota: Elaboración propia. 


\section{VIÑAS-MARTE • GUZMÁN-TAVERAS}

Análisis de los recursos para la enseñanza de lectoescritura inicial a la luz del currículo dominicano

\section{Resultados}

En este apartado se presentan los hallazgos a partir de las dimensiones analizadas de cada recurso que conformó la muestra. Se presentan las categorías y su presencia en los recursos de la base LEI-RD, según el tipo de institución que los produjo, además de una descripción de los hallazgos más relevantes del estudio. Los resultados se presentan a partir de la muestra seleccionada de 115 recursos pedagógicos de un total de 485 presentes en dicha base.

\subsection{Finalidad del recurso}

Los recursos de LEI-RD van dirigidos a grupos específicos de población (niños de 6 a 9 años, docentes, acompañantes de docentes, y padres, entre otros) los cuales desarrollarán la habilidad o competencia a la que está destinado cada recurso, tomando en cuenta su contenido y lo que se describe en la base de datos. Los resultados relativos a esta dimensión muestran que el $75.60 \%$ tiene como finalidad la enseñanza de la lectura y de la escritura, frente a un $7 \%$ dedicado al aprendizaje (tabla 6).

Tabla 6. Finalidad de los recursos de la base de datos LEI-RD por tipo de institución

\begin{tabular}{|c|c|c|c|c|c|c|c|c|}
\hline \multicolumn{9}{|c|}{ Dimensión: finalidad del recurso } \\
\hline \multirow[b]{2}{*}{ Categorías } & \multicolumn{4}{|c|}{ FRE } & \multicolumn{4}{|c|}{$\%$} \\
\hline & $\begin{array}{c}\text { Total } \\
n=115\end{array}$ & PUB & PRI & ISFL & Total & PUB & PRI & ISFL \\
\hline Enseñanza de la lectura & 51 & 17 & 20 & 14 & 44.35 & 14.78 & 17.39 & 12.17 \\
\hline Aprendizaje de la lectura & 7 & 2 & 2 & 3 & 6.09 & 1.74 & 1.74 & 2.61 \\
\hline Enseñanza de la escritura & 36 & 17 & 13 & 6 & 31.30 & 14.78 & 11.30 & 5.22 \\
\hline Aprendizaje de la escritura & 1 & 0 & 1 & 0 & 0.87 & 0.00 & 0.87 & 0.00 \\
\hline Evaluación diagnóstica & 13 & 9 & 4 & 0 & 11.30 & 7.83 & 3.48 & 0.00 \\
\hline Evaluación formativa & 13 & 8 & 3 & 2 & 11.30 & 6.96 & 2.61 & 1.74 \\
\hline Evaluación sumativa & 0 & 0 & 0 & 0 & 0.00 & 0.00 & 0.00 & 0.00 \\
\hline Formación docente & 19 & 3 & 12 & 4 & 16.52 & 2.61 & 10.43 & 3.48 \\
\hline Acompañamiento pedagógico & 7 & 0 & 6 & 1 & 6.09 & 0.00 & 5.22 & 0.87 \\
\hline
\end{tabular}

Nota: Elaboración propia a partir de la base de datos LEI-RD. $n=115$. FRE: Frecuencia; PUB: Pública; PRI: Privada; ISFL: Institución sin fines de lucro. Hay recursos en los que se evidenció más de una categoría, por lo que fueron codificados más de una vez; por tal razón, al sumar los porcentajes generales, el total excede 100 .

\subsection{Habilidades de la lectoescritura}

El currículo dominicano propicia el abordaje de habilidades de LEI a partir del texto mediante los contenidos conceptuales, procedimentales y actitudinales. Las competencias más presentes son la comprensión y producción oral y la comprensión y producción escrita, así como la decodificación. Por otra parte, la conciencia fonológica, el vocabulario, las etapas de apropiación y el dominio del código (transcripción, caligrafía) afloran escasamente en los recursos estudiados. La habilidad que más se evidencia en los recursos producidos por los tres tipos de instituciones (públicas, privadas e ISFL) es la comprensión escrita, con porcentajes del $23.50 \%, 21.70 \%$ y $10.40 \%$, respectivamente. Por el contrario, la habilidad menos presente corresponde a las etapas de apropiación de la escritura, con un porcentaje de $0.90 \%, 0.00 \%$ y $2.60 \%$ por tipo de institución (Tabla 7 ). 
Revista Caribeña de Investigación Educativa | 2020, 4(2), 96-111

Tabla 7. Habilidades de la lectoescritura evidenciadas en los recursos de LEI-RD por tipo de institución

\begin{tabular}{|c|c|c|c|c|c|c|c|c|}
\hline \multicolumn{9}{|c|}{ Dimensión: habilidades de lectoescritura } \\
\hline \multirow[b]{2}{*}{ Categorías } & \multicolumn{4}{|c|}{ FRE } & \multicolumn{4}{|c|}{$\%$} \\
\hline & $\begin{array}{c}\text { Total } \\
n=115\end{array}$ & PUB & PRI & ISFL & Total & PUB & PRI & ISFL \\
\hline Comprensión oral & 33 & 15 & 15 & 3 & 28.70 & 13.04 & 13.04 & 2.61 \\
\hline Producción oral & 28 & 15 & 10 & 3 & 24.35 & 13.04 & 8.70 & 2.61 \\
\hline Conciencia fonológica & 13 & 1 & 9 & 3 & 11.30 & 0.87 & 7.83 & 2.61 \\
\hline Comprensión escrita & 64 & 27 & 25 & 12 & 55.65 & 23.48 & 21.74 & 10.43 \\
\hline Convenciones de la lectoescritura & 26 & 11 & 10 & 5 & 22.61 & 9.57 & 8.70 & 4.35 \\
\hline Decodificación & 28 & 12 & 11 & 5 & 24.35 & 10.43 & 9.57 & 4.35 \\
\hline Vocabulario & 20 & 5 & 13 & 2 & 17.39 & 4.35 & 11.30 & 1.74 \\
\hline Fluidez lectora & 16 & 9 & 4 & 3 & 13.91 & 7.83 & 3.48 & 2.61 \\
\hline Producción escrita & 54 & 25 & 18 & 11 & 46.96 & 21.74 & 15.65 & 9.57 \\
\hline $\begin{array}{l}\text { Etapas de apropiación de la escri- } \\
\text { tura }\end{array}$ & 4 & 1 & 0 & 3 & 3.48 & 0.87 & 0.00 & 2.61 \\
\hline $\begin{array}{l}\text { Dominio del código escrito (Trans- } \\
\text { cripción, Caligrafía) }\end{array}$ & 19 & 13 & 6 & 0 & 16.52 & 11.30 & 5.22 & 0.00 \\
\hline
\end{tabular}

Nota: Elaboración propia a partir de la base de datos LEI-RD. $n=115$. FRE: Frecuencia; PUB: Pública; PRI: Privada; ISFL: Institución sin fines de lucro. Hay recursos en los que se evidenció más de una categoría, por lo que fueron codificados más de una vez; por tal razón, al sumar los porcentajes generales el total excede de 100 .

\subsection{Enfoques de enseñanza}

Se identificaron varios enfoques. Alrededor del $81 \%$ de los recursos evidencia puntos de encuentro con los enfoques y teorías que sirven de base al currículo dominicano, donde predomina el enfoque textual, funcional y comunicativo con un $40 \%$. Tiene menor presencia, con un $11.30 \%$, el enfoque por destrezas aisladas. El $38.30 \%$ de los recursos analizados procedentes de las instituciones públicas coincide con los enfoques propuestos por el currículo, y superan ligeramente a las instituciones privadas cuyos recursos son coherentes con dicho documento en un $29.60 \%$ (Tabla 8).

Tabla 8. Enfoques de enseńanza de los recursos según tipo de institución

\section{Dimensión: enfoques de enseñanza}

\begin{tabular}{lcccccccc}
\hline \multirow{2}{*}{ Categorías } & \multicolumn{4}{c}{ FRE } & & \multicolumn{3}{c}{$\%$} \\
\cline { 2 - 8 } & $\begin{array}{c}\text { Total } \\
\mathbf{n = 1 1 5}\end{array}$ & PUB & PRI & ISFL & Total & PUB & PRI & ISFL \\
\hline Textual, funcional y comunicativo & 46 & 20 & 21 & 5 & 40.00 & 17.39 & 18.26 & 4.35 \\
\hline Procesual cognitivo, por competencias & 26 & 13 & 8 & 5 & 22.61 & 11.30 & 6.96 & 4.35 \\
\hline Sociocultural & 21 & 11 & 5 & 5 & 18.26 & 9.57 & 4.35 & 4.35 \\
\hline Por destrezas aisladas & 13 & 6 & 6 & 1 & 11.30 & 5.22 & 5.22 & 0.87 \\
\hline
\end{tabular}

Nota: Elaboración propia a partir de la base de datos LEI-RD. $n=115$. FRE: Frecuencia; PUB: Pública; PRI: Privada; ISFL: Institución sin fines de lucro. 


\subsection{Animación lectora}

La animación de la lectura se refiere a las acciones dirigidas a promover el disfrute, más allá de su función epistémica propia de ambientes académicos. Los resultados concernientes a esta dimensión evidencian escasa presencia de sus categorías en los recursos ana- lizados. El 26.10\% hace referencia a la animación de la lectura. De este porcentaje, los recursos procedentes de las instituciones sin fines de lucro representan el $16.40 \%$, por encima de las instituciones públicas y privadas (Tabla 9).

Tabla 9. Frecuencia de recursos de LEI-RD que promueven la animación lectora por tipo de institución

\begin{tabular}{lcccccccc}
\hline \multicolumn{1}{c}{ Dimensión: animación lectora } \\
\hline \multirow{2}{*}{ Categorías } & \multicolumn{1}{c}{ FRE } & \multicolumn{7}{c}{ \% } \\
\cline { 2 - 11 } & $\begin{array}{c}\text { Total } \\
n=115\end{array}$ & PUB & PRI & ISFL & Total & PUB & PRI & ISFL \\
\hline Ambiente letrado & 6 & 1 & 3 & 2 & 5.22 & 0.87 & 2.61 & 1.74 \\
\hline Promoción del hábito lector & 7 & 1 & 0 & 6 & 6.09 & 0.87 & 0.00 & 5.22 \\
\hline La biblioteca áulica y escolar & 8 & 2 & 1 & 5 & 6.96 & 1.74 & 0.87 & 4.35 \\
\hline Familia y hábito lector & 4 & 0 & 1 & 3 & 3.48 & 0.00 & 0.87 & 2.61 \\
\hline Escuela y hábito lector & 5 & 0 & 2 & 3 & 4.35 & 0.00 & 1.74 & 2.61 \\
\hline
\end{tabular}

Nota: Elaboración propia a partir de la base de datos LEI-RD. $n=115$. FRE: Frecuencia; PUB: Pública; PRI: Privada; ISFL: Institución sin fines de lucro.

\subsection{Pertinencia del recurso}

Con relación a la pertinencia o no de los recursos de la base de datos LEI-RD, los resultados mostraron que el $38.26 \%$ se ubica en el nivel satisfactorio y el
$39.13 \%$ en medianamente satisfactorio, y las instituciones privadas quedan ligeramente por encima de las públicas. Por el contrario, el $22.34 \%$ se ubica en los niveles que corresponden a área de oportunidad y no satisfactorio (Tabla 10).

Tabla 10. Pertinencia de los recursos LEI-RD según la escala de valoración y el tipo de institución

\begin{tabular}{lccccccccc}
\hline \multicolumn{1}{c}{ Pertinencia de los recursos } \\
\hline \multirow{2}{*}{ Categorías } & \multicolumn{1}{c}{ FRE } & \multicolumn{1}{c}{$\%$} \\
\cline { 2 - 11 } & $\begin{array}{c}\text { Total } \\
(\boldsymbol{n}=\mathbf{1 1 5})\end{array}$ & PUB & PRI & ISFL & Total & PUB & PRI & ISFL \\
\hline Satisfactorio & 44 & 18 & 16 & 10 & 38.26 & 15.65 & 13.91 & 8.70 \\
\hline Medianamente satisfactorio & 45 & 16 & 23 & 6 & 39.13 & 13.91 & 20.00 & 5.22. \\
\hline Área de oportunidad & 18 & 11 & 2 & 5 & 15.65 & 9.57 & 1.74 & 4.35 \\
\hline No satisfactorio & 8 & 3 & 4 & 1 & 6.96 & 2.61 & 3.48 & 0.87 \\
\hline
\end{tabular}

Nota: Elaboración propia a partir de la base de datos LEI-RD. $n=115$ : FRE: Frecuencia; PUB: Pública; PRI: Privada; ISFL: Institución sin fines de lucro. 


\section{Discusión y conclusiones}

Sobre la primera pregunta de investigación ¿Cómo se vinculan los recursos de LEI-RD con el currículo del primer ciclo de primaria?, los resultados muestran que en las habilidades de LEI que promueven los recursos, predomina la comprensión escrita y, en menor medida, la comprensión y producción oral. Según las evidencias (Ferreiro \& Teberosky, 1991; Limpo \& Alves, 2013; Shanahan, 2005) para el desarrollo de estas competencias se requiere la adquisición de habilidades como la conciencia fonológica, el vocabulario, la transcripción y la reflexión sobre el proceso de apropiación de la escritura. Sin embargo, los resultados evidencian que en los recursos son delegadas a un segundo plano, al ser abordadas de manera superficial, sin considerar todos los subprocesos que inciden en su desarrollo, a pesar de que el currículo dominicano establece la integración de cada uno.

La conciencia fonológica y las etapas de escritura han sido los aspectos menos trabajados; los recursos que promueven estas habilidades se encuentran en las instituciones privadas e internacionales. Este resultado es preocupante, ya que evidencia falta de coherencia del sector público con sus propios lineamientos curriculares. Esta carencia puede provocar dificultades en el aprendizaje de la LEI y, en consecuencia, en el desarrollo del pensamiento. Una situación similar se refleja en el estudio realizado por Stone (2019), en el que se establece que existe una brecha entre el desarrollo de habilidades de preescritura y escritura y los resultados de la LEI. En este sentido, resulta imperativo que los actores clave estudien la inclusión de intervenciones que favorezcan el desarrollo de la conciencia fonológica, la decodificación, la fluidez lectora, el vocabulario y los niveles de procesamiento escritor (transcripción, caligrafía, etc.) en sus recursos pedagógicos, dirigidos tanto a la enseńanza como al aprendizaje, para así alinearlos con los planteamientos curriculares.

Sobre la segunda pregunta de investigación ¿Cuál es el enfoque de enseñanza que predomina en el contenido de los recursos pedagógicos incluidos en la base de datos de lectoescritura inicial LEI-RD?, en los recursos pedagógicos de LEI-RD se observan puntos de encuentro con los enfoques propuestos por el currículo dominicano, los cuales enfatizan en el aprendizaje, amparados en el constructivismo. Los resultados relacionados con este aspecto muestran que el enfoque textual, funcional y comunicativo es el que cuenta con mayor presencia, seguido del procesual cognitivo, por competencias. Sin embargo, varias investigaciones previas concluyen que los docentes centran su atención en la enseñanza, a pesar de que confiesan basar sus intervenciones en la teoría constructivista en la que adquieren mayor importancia los enfoques centrados en el aprendizaje (De Lima, 2018). Resulta necesario que se realicen futuras investigaciones para constatar la manera en que se utilizan los recursos pedagógicos durante el proceso de enseńanza aprendizaje, en el que el uso de estos recursos no siempre está alineado con los enfoques propuestos en el currículo.

La dimensión de animación o promoción de la lectura constituye una acción de intervención sociocultural que impulsa la reflexión, revalorización y construcción de nuevos sentidos, idearios y prácticas lectoras (Álvarez \& Naranjo, 2003, citado en Higuera Guarín, 2016). El currículo dominicano promueve la creación de ambientes alfabetizadores y el uso de bibliotecas. Sin embargo, en los recursos no se evidencian propuestas destinadas a estimular la lectura por placer y la integración de la familia en la formación del hábito lector. Esta necesidad guarda correspondencia con los resultados obtenidos, los cuales muestran que son escasos los recursos de instituciones públicas y privadas que van dirigidos a este objetivo. Estos hallazgos coinciden con investigaciones internacionales sobre el tema (Lluch \& Sánchez-García, 2017) que subrayan la necesidad de promover el hábito lector desde la primera infancia. En consecuencia, la falta de iniciativas que incentiven el amor por la lectura puede generar apatía o rechazo. Por tanto, se sugiere tomar en cuenta la inclusión de estrategias de animación a la lectura e integración de la familia en la creación de materiales pedagógicos que apoyen la implementación del currículo vigente. 


\section{VIÑAS-MARTE • GUZMÁN-TAVERAS}

Análisis de los recursos para la enseñanza de lectoescritura inicial a la luz del currículo dominicano

La investigación también identifica áreas de mejora de la base LEI-RD. Los resultados muestran que la mayoría de los recursos se califican en un nivel satisfactorio o medianamente satisfactorio de acuerdo con el vínculo que guardan con los enfoques que promueve el currículo dominicano y las evidencias actuales. Sin embargo, el $22.61 \%$ de los recursos presenta áreas de oportunidad, es decir, promueven intervenciones pedagógicas desvinculadas de lo prescripto en el currículo y en la literatura consultada o eluden aspectos relevantes para la adquisición de competencias comunicativas. Un gran número de estos recursos pone énfasis en el sistema de escritura y obvia los niveles altos del proceso de lectura (comprensión inferencial, analítica y crítica) así como los de escritura (planificación, textualización y revisión), cuya incidencia fue destacada por Limpo y Alves (2013). Por otro lado, de este porcentaje una cantidad considerable de recursos de LEI-RD carece de actividades o consignas que orienten su aplicación en el aula, ya que se trata de textos, canciones, imágenes, fichas bibliográficas, enlaces expirados o documentos no descargables que no responden al objetivo de este repositorio. Por tanto, los resultados de este estudio clasificaron dichos recursos como no satisfactorios.

Según el protocolo establecido por el Recolector de Ciencia Abierta (RECOLECTA), el contenido de acceso restringido disponible en un repositorio no debe exceder el $25 \%$. La base de datos LEI-RD contiene 351 recursos de acceso restringido sobre un total de 948, eso representa un $37.42 \%$, valor que está por encima de lo recomendado (Azorín-Millaruelo et al., 2017). Esto sugiere el establecimiento de criterios relacionados con las dimensiones estudiadas para la inclusión de nuevos recursos, así como la reorientación de los existentes. De este modo, el contenido presentado a los usuarios (docentes, investigadores y personas interesadas) respondería, con más aciertos, a sus necesidades. Por su parte, el instrumento utilizado durante esta investigación para evaluar la pertinencia de los recursos presentes en la muestra está basado en las evidencias consultadas y podría ser considerado como un aporte para la valoración del contenido restante de esta base y de otras de la región.
En resumen, esta investigación demostró que muchos de los recursos presentes en la base de datos LEI-RD no abordan subprocesos de lectura y escritura ni habilidades necesarias para desarrollar de manera integral las competencias comunicativas que propone el currículo dominicano, a pesar de que se observan intervenciones pedagógicas propias del enfoque textual, funcional y comunicativo, así como del procesual cognitivo, por competencias. En consecuencia, se sugiere la exclusión de los recursos de LEI-RD que presentan áreas de oportunidad y los clasificados como no satisfactorios, ya que evidencian cierta desvinculación con el currículo nacional y no se corresponden con la finalidad de dicha base. También se recomienda incluir otros modos de organización de la estructura de la base, con opciones relativas a las dimensiones y categorías establecidas en esta investigación, especialmente de libre acceso. Por último, se sugiere incorporar más recursos alineados al currículo y a sus recomendaciones para la lectoescritura para garantizar un mejor uso de dichos recursos, y que aporten a la mejora de la enseñanza aprendizaje de la lectoescritura inicial.

Es importante mencionar las limitaciones de esta investigación, entre ellas, el tiempo de duración de la beca obtenida para la realización del estudio, lo que no permitió la exploración de todos los recursos contenidos en LEI-RD. Tampoco se pudo elaborar un instrumento de evaluación por dimensión que permitiera analizar de manera más profunda y detallada todos los recursos. Estos aspectos deben considerarse para futuras investigaciones que complementen los hallazgos de este estudio.

\section{Agradecimientos}

Esta investigación se realizó con los fondos de la Red para la Lectoescritura Inicial de Centroamérica y el Caribe (RedLEI). Los autores expresan gratitud por la formación y el acompañamiento recibido de la RedLEI durante el proceso de investigación. También agradecen el asesoramiento de Carmen García, directora del Centro de Excelencia para la Difusión e Investigación de la Lectura y Escritura (CEDILE) de la Pontificia Universidad Católica Madre y Maestra, universidad perteneciente a la Red. 


\section{Referencias bibliográficas}

Azorín-Millaruelo, C., Bernal-Martínez, I., Cívico-Martín, R., Cózar-Santiago, A., Guzmán-Pérez, C., Losada-Yáñez, M., Morillo-Moreno, J., Nonó-Rius, B., Padrós-Cuxart, R., \& Prats-Prat, J. (2017). Guía para la evaluación de repositorios institucionales de investigación. Espańa: Recolecta.

Blanco, L., Cuter, M. E., Grúnfeld, D., Rodríguez, M. E., \& Calmels, D. (2007). Enseñar lengua en la Escuela Primaria. Argentina: Tinta fresca Ediciones.

Charria, M., \& González, A. (1992). Hacia una nueva pedagogía de la lectura, Argentina: AIQUE Educación.

National Reading Panel, National Institute of Child Health and Human Development. (2000). Report of the National Reading Panel: Teaching children to read: An evidence-based assessment of the scientific research literature on reading and its implications for reading instruction: Reports of the subgroups. National Institutes of Health. http://r.issu.edu.do/l.php?l=78tEP

Daviña, L. (2003). Adquisición de la Lectoescritura. Revisión critica de métodos y teorías. Santa Fe, Argentina: Homo Sapiens.

De Lima, D., \& Montenegro, L. (2010). Las guías de lectura y escritura y su importancia para la enseñanza-aprendizaje de la lengua en contextos socioeconómicos vulnerables. En D. R. ILCE (coord.), Aprendizaje efectivo de la lectoescritura (pp. 43-61). México: ILCE.

De Lima, D. (2018). Teoría de aprendizaje y práctica alfabetizadora en los primeros grados de primaria. Santo Domingo: IDEICE.

Ferreiro, E., \& Teberosky, A. (1991). Los sistemas de escritura en el desarrollo del niño. Argentina: Siglo XXI editores.

Ferreiro, E. (2006). La escritura antes de la letra. CPU-e, Revista de Investigación Educativa, 3, 1-52. http://r.issu.edu.do/l.php?l=79aKd

Fons, M. (2004). Leer y escribir para vivir. Alfabetiza- ción inicial y uso real de la lengua escrita en la escuela. Barcelona: Editorial Graó.

Gutiérrez, F., \& Díez, A. (2018). Conciencia fonológica y desarrollo evolutivo de la escritura en las primeras edades. Educación XXI, 21(1), 395-415. http://r.issu.edu.do/l.php?l=81y9H

Higuera Guarín (2016). De la estética de la recepción a la animación a la lectura: consideraciones teóricas para una propuesta de animación de la lectura literaria en espacios no convencionales. La Palabra, (28), 187-200. https://doi.org/10.19053/01218530.4815

Kaufman, A. (coord.) (2009). Leer y escribir: el dia a día en las aulas, Buenos Aires: AIQUE Educación.

Limpo, T., \& Alves, R. A. (2013). Modeling writing development: Contribution of transcription and self-regulation to Portuguese students' text generation quality. Journal of Educational Psychology, 105(2), 401-413. https://doi.org/10.1037/a0031391

Lluch, G., \& Sánchez-García, S. (2017). La promoción de la lectura: un análisis crítico de los artículos de investigación, Revista Española de Documentación Cientifica, 40(4), 114.

http://dx.doi.org/10.3989/redc.2017.4.1450

Ministerio de Educación de Argentina. (2010). La formación docente en alfabetización inicial.

http://r.issu.edu.do/l.php?l=82vwf

Ministerio de Educación de la República Dominicana (MINERD). (2016). Diseño curricular. Nivel Primario, Primer Ciclo. República Dominicana: Ministerio de Educación.

Ministerio de Educación de la República Dominicana (MINERD). (2017). Resultados de la evaluación diagnóstica nacional de tercer grado de primaria. Santo Domingo: MINERD.

Ministerio de Educación República de Chile. (2011). Estándares para las bibliotecas escolares CRA.

http://r.issu.edu.do/l.php?l=83MGz

Montenegro, L. (2013). Proyecto: Revisión y actualización del currículo vigente.

http://r.issu.edu.do/l.php?l=84s8a 


\section{VIÑAS-MARTE • GUZMÁN-TAVERAS}

Análisis de los recursos para la enseñanza de lectoescritura inicial a la luz del currículo dominicano

Montenegro, L. (2010). El enfoque comunicativo de la enseñanza de la lengua. En Coordinación General de Investigación y Desarrollo de Modelos Educativos y del Instituto Latinoamericano de la Comunicación Educativa (ILCE) (Eds.), Aprendizaje efectivo de la lectoescritura: Una experiencia regional exitosa (pp. 43-61). México: ILCE.

Ortiz, D., \& Robino, A. (2003). Cómo se aprende, cómo se enseña la lengua escrita. Buenos Aires: Lugar Editorial.

Rasinski, T. (2010). The Fluent Reader. New York: Scholastic.

Romero, L. (2014). El aprendizaje de la lectoescritura. Nicaragua: Fe y Alegría.

Rosemberg, C., Menti, A., Stein, A., Alam, F., \& Migdalek, M. (2016). Vocabulario, narración y argumentación en los primeros años de la infancia y la niñez. Revista Costarricense de Psicología, 35(2), 139158. http://r.issu.edu.do/l.php?l=224lq1
Shanahann, T. (2005). The National Reading Panel Report: Practical Advice for teachers. Chicago: Learning Point Associates.

Stone, R. (2019). Lectoescritura inicial en América Latina y el Caribe: una revisión sistemática. Revista de Investigación y Evaluación Educativa, 6(1), 22-36. http://r.issu.edu.do/l.php?l=86Vge

Teberosky, A. (2000, diciembre). Los sistemas de escritura. Trabajo presentado en Congreso Mundial de Lectoescritura, Universidad de Barcelona, Barcelona.

Vijil, J. (2019). Actores clave en lectoescritura inicial en República Dominicana. Resultados de un mapeo y análisis de actores, Revista de Investigación y Evaluación Educativa, 6, 38-49.

http://r.issu.edu.do/l.php?l=230gP8

Yepes, L. B., Caretta, M. G., \& Díez, C. (2013). Jóvenes lectores. Caminos de Formación. Montevideo, Uruguay: Ministerio de Educación y Cultura.

\section{CÓMO CITAR:}

Viñas-Marte, R., \& Guzmán-Taveras, L. N. (2020). Análisis de los recursos para la enseñanza de lectoescritura inicial a la luz del currículo dominicano. Revista Caribeña de Investigación Educativa (RECIE), 4(2), 96-111. https://doi.org/10.32541/recie.2020. v4i2.pp96-111 